\title{
Measurement of Ultrashort Vector Pulses From Polarization Gates by In-Line, Single-Channel Spectral Interferometry
}

\author{
Benjamín Alonso ${ }^{(1)}$ and Íñigo J. Sola
}

\begin{abstract}
The growing use of ultrashort laser pulses exhibiting time-varying polarization (vector pulses) demands simple and robust characterization techniques capable to perform measurements in a broad range of experimental and environmental conditions. Here, we present in-line, single-channel setup based on spectral interferometry to characterize ultrashort vector pulses. The use of a bulk interferometer based on birefringence is key for the stability and sensitivity of the technique, thus being simple and highly robust. The technique is used to measure vector pulses corresponding to polarization gates, which are used in many applications. Those results are validated by simulations. The technique here presented has a number of potential applications in nonlinear effects (e.g., transient birefringence and nonlinear phenomena with vector pulses).
\end{abstract}

Index Terms-Ultrafast lasers, ultrashort pulses, optical polarization measurements, vector pulses, polarization gate.

\section{INTRODUCTION}

A DVANCES on laser technology are enabling the generation and use of pulses of light more and more complex, both on time (e.g., single-cycle pulses) and singular spatial distributions (e.g., ultrashort pulsed vortex, Bessel beams, etc.). In addition, there is a raising interest on ultrashort pulses exhibiting time-varying polarization (vector pulses) [1]-[4]. The use and analysis of vector pulses is a useful tool in the study of quantum wells properties [5], vector coherent control for selective isomerization of enantiomers [6]-[9] and the generation of $\mathrm{THz}$ pulses with time evolving polarization from IR vector pulses [7], to mention some examples.

Manuscript received October 23, 2018; revised February 14, 2019; accepted March 11, 2019. This work was supported in part by Junta de Castilla y León under Grant SA287P18, Spanish MINECO under Grants FIS2017-87970-R and EQC2018-004117-P, and the European Union's Horizon 2020 Research and Innovation Programme under the Marie Sklodowska-Curie Individual Fellowship under Grant 798264. (Corresponding author: Benjamín Alonso.)

B. Alonso was with the Grupo de Investigación en Aplicaciones del Láser y Fotónica, Departamento de Física Aplicada, Universidad de Salamanca, Salamanca E-37008, Spain. He is now with the Sphere Ultrafast Photonics, S.A., Parque de Ciência e Tecnologia da Universidade do Porto, 4169-007 Porto, Portugal (e-mail: b.alonso@usal.es; ijsola@usal.es).

I. J. Sola is with the Grupo de Investigación en Aplicaciones del Láser y Fotónica, Departamento de Física Aplicada, Universidad de Salamanca, Salamanca E-37008, Spain

Color versions of one or more of the figures in this paper are available online at http://ieeexplore.ieee.org.

Digital Object Identifier 10.1109/JSTQE.2019.2906266
In the attoscience field, vector pulses are used to alter the high-order harmonic generation (HHG) process in gas. Because of the high sensitivity of the HHG on atoms to light polarization (when light polarization is not linear, HHG efficiency vanishes), ultrashort pulses with particular temporal evolving polarization, known as polarization gates (PG), are used to control the number of attosecond light bursts created in the process [10]-[12]. When applied to few-cycle pulses, the resulting vector pulses from the PG are able to generate isolated attosecond pulses through HHG [13]. In a different configuration, two noncollinear counter-rotating circularly polarized beams create a vector pulse that generates isolated circularly polarized attosecond pulses by HHG [14].

In this context, the characterization of the time-dependent polarization of the pulse is a key point. The reconstruction of ultrashort pulses has been developed since decades. Nowadays, techniques such as FROG [15], SPIDER [16] or d-scan [17], are well established and allow to measure pulses down to few optical cycles and single-cycle regime [18]-[22]. However, the usual techniques are designed for scalar pulses where pulse polarization is linear and constant on time.

During the last years, different approaches have been proposed to accomplish vector pulse reconstruction in the femtosecond range, e.g., through spectral interferometry in different configurations [23], [24], tomographic reconstruction from measurement of different projections [25], time-resolved ellipsometry [26], some variants [27], [28] based on X-FROG technique [29], nanointerferometric measurement of vector beams [30], or spatial-spectral interferometry [31].

In particular, in dual-channel spectral interferometry method, known as POLLIWOG [21], a linearly polarized known reference pulse interferes with a delayed test (unknown) pulse. By using spectral interferometry analysis [32], the relative phase between two polarization components of the test pulse is extracted. Together with the amplitudes for each polarization projection and the spectral phase of the reference pulse (measured with any scalar temporal pulse reconstruction technique), the time-dependent polarization pulses can be reconstructed. The use of a linear process as interferometry makes it a sensitive and powerful technique, with a non-iterative retrieval algorithm. However, precisely because of this interferometric nature, typical schemes commonly based on standard interferometers such as Mach-Zehnder or Michelson will be very demanding 
on stability, in order to preserve the spectral fringes and, more important, to accurately measure the phase difference between the orthogonal components. In fact, the POLLIWOG uses a dual-channel spectrometer to record separately and simultaneously the interferences of the $\mathrm{x}$ and $\mathrm{y}$-components, thus preventing shot-to-shot phase fluctuations to affect the polarization component relative phase measurements.

Therefore, it results that, to determine the time-varying polarization state, it is a key point the accurate measurement of the relative amplitude and, primarily, the phase of the polarization component. In this context, to fulfill the stability requirements, our proposal is based on an in-line single-channel spectral interferometry setup, in which the relative phase is directly measured in a crossed intermediate angle projection. The objective of the present work is to implement a robust and reliable technique, not affected by instabilities and noise, and simple in order to be applied to different experimental cases, e.g., PGs that are used for different applications.

Firstly, we present the description of the method and the set-up used for the experimental implementation, accompanied with a first experimental validation of a vector pulse and a study of stability. Secondly, we apply it to the reconstruction of vector pulses obtained from PG technique. All measurements are corroborated by simulations. Finally, we summarize the conclusions.

\section{The Method: IN-Line SPeCtRAL INTERFEROMETRY}

Our method takes the advantages of using interferometry, a process characterized by being linear, high sensitivity or a direct analysis, while avoiding the main drawback, i.e., the instabilities and alignment requirements. Mostly, spectral interferometry set-ups are based on interferometers, where the unknown pulse is combined with the one serving as a reference. These setups are very sensitive to vibrations and instabilities from the environment. To prevent this problem, we use a birefringent element as a monolithic in-line interferometer, a calcite plate $(3 \mathrm{~mm}$ of thickness), with their optical axes parallel to the input and output faces. When an incoming vector pulse passes through the plate, it is split into the ordinary and extraordinary components, presenting a delay due to the different refractive indices of the birefringent material (in our case, $1.8 \mathrm{ps}$ ).

In previous works, birefringent crystals have already been used to create stable delayed pulse replicas [33]-[35], which has been applied to measure the spectral phase of scalar pulses [34]-[37].

After the calcite plate, we use a linear polarizer (LP) to select different polarization projections of the delayed components and the corresponding spectra are subsequently acquired by a spectrometer (AvaSpec 2048-USB1, from Avantes Inc.). Just by varying the LP orientation, it is possible to measure the spectra from the ordinary and extraordinary components respectively and the interference between both at an intermediate angle. This latter spectrum encodes the spectral phase difference among the ordinary and extraordinary components (once the phase added by the birefringent plate is subtracted). Therefore, a vector component of the pulse is actually acting as a reference for the orthogonal one. The use of a bulk interferometer means that both components travel through the same physical path and there is 131 not any other pulse splitting or recombination, thus awarding 132 the pursued stability to the detection, as well as getting rid of 133 a precise alignment. Finally, if one of the components is char- 134 acterized and its spectral phase is known (e.g., by means of a 135 standard scalar temporal pulse reconstruction technique), it is 136 possible to reconstruct the vector pulse by applying Fourier- 137 transform spectral interferometry analysis (FTSI) [32] to the 138 intermediate projection. 139

The experimental setup and the axes convention are rep- 140 resented in Fig. 1. Rotation angles for the different elements 141 will be given with respect to the $\mathrm{X}$-axis and being the observer 142 looking to the source (positive rotation angle means counter- 143 clockwise). Firstly, the unknown vector pulse impinges the bire- 144 fringent plate (calcite $3 \mathrm{~mm}$ thickness, 0.5 "-side square) that is 145 located with the extraordinary (fast) axis horizontal (x-axis), 146 being the y-component of the pulse delayed with respect to 147 the X-component. The LP can be situated at different angles 148 $\alpha$, selecting the corresponding pulse projections, $S_{\alpha}$, that are 149 detected in the fiber coupled spectrometer.

To calibrate in amplitude and phase the system, we create a 151 calibrating pulse linearly polarized at $45^{\circ}$. This pulse has the 152 same spectral amplitudes $A_{y}(\omega)=A_{x}(\omega)$ and phases $\phi_{y}(\omega)=153$ $\phi_{x}(\omega)$, so we can obtain the amplitude response of the system 154 and the relative dispersion of the calcite axes.

The horizontal and vertical projections of the LP provide the 156 information of the amplitude of the X-and y-components, with 157 $S_{0^{\circ}}=S_{x}$ and $S_{90^{\circ}}=S_{y}$ respectively. The intermediate projec- 158 tion at $\alpha=45^{\circ}$ encodes the relative phase between the orthog- 159 onal components ( $\mathrm{x}$ and $\mathrm{y}$ ):

$$
S_{45^{\circ}}=1 / 2 S_{x}+1 / 2 S_{y}+\sqrt{S_{x} S_{y}} \cos \left[\left(\phi_{y}-\phi_{x}\right)_{\text {meas }}\right] .
$$

This relative phase gathers the pulse phase and the calcite 161 phase, $\left(\phi_{y}-\phi_{x}\right)_{\text {meas }}=\left(\phi_{y}-\phi_{x}\right)_{\text {pulse }}+\left(\phi_{y}-\phi_{x}\right)_{\text {calc }}$, be- 162 ing the calcite contribution the one that introduces the de- 163 lay required to use the FTSI algorithm. In order to remove 164 the calcite relative dispersion, we used the projection $S_{45^{\circ}} 165$ of the calibrating pulse, for which $\left(\phi_{y}-\phi_{x}\right)_{\text {pulse }}^{[\text {Calibr }]}=0$ and 166 $\left(\phi_{y}-\phi_{x}\right)_{\text {meas }}^{[\text {Calibr }]}=\left(\phi_{y}-\phi_{x}\right)_{\text {calc }}$.

Regarding the amplitude sensitivity of the fiber coupled 168 spectrometer to the $\mathrm{x}$ and $\mathrm{y}$ components, it is corrected with 169 the $0^{\circ}$ and $90^{\circ}$ projections of the same calibrating pulse 170 $\left(S_{y}=S_{x}\right)$. The y-component of the pulse to be measured is 171 corrected as $S_{y}{ }^{[P u l s e]}=S_{y}{ }^{[P u l s e]} R_{\text {calibr }}$, where $R_{\text {calibr }}(\omega)=172$ $S_{y}{ }^{[\text {Calibr }]} / S_{x}{ }^{[\text {Calibr }]}$ is smoothed and interpolated within the 173 spectral range of the calibrating pulse.

The spectral phase of the component acting as a reference, in 175 our case the X-component, is measured with a SPIDER device 176 (any temporal characterization technique is valid). With the LP 177 set horizontal and a flip mirror, we extract this reference pulse. 178 Under this configuration, the $\mathrm{x}$-axis dispersion of the calcite 179 [38] needs to be subtracted (alternatively, the projection of this 180 reference pulse can be extracted before the calcite). 181

The proposed scheme requires that both the $\mathrm{x}-$ and $\mathrm{y}-182$ components of the pulse have similar spectral content and 183 comparable amplitude in order to obtain suitable spectral 184 


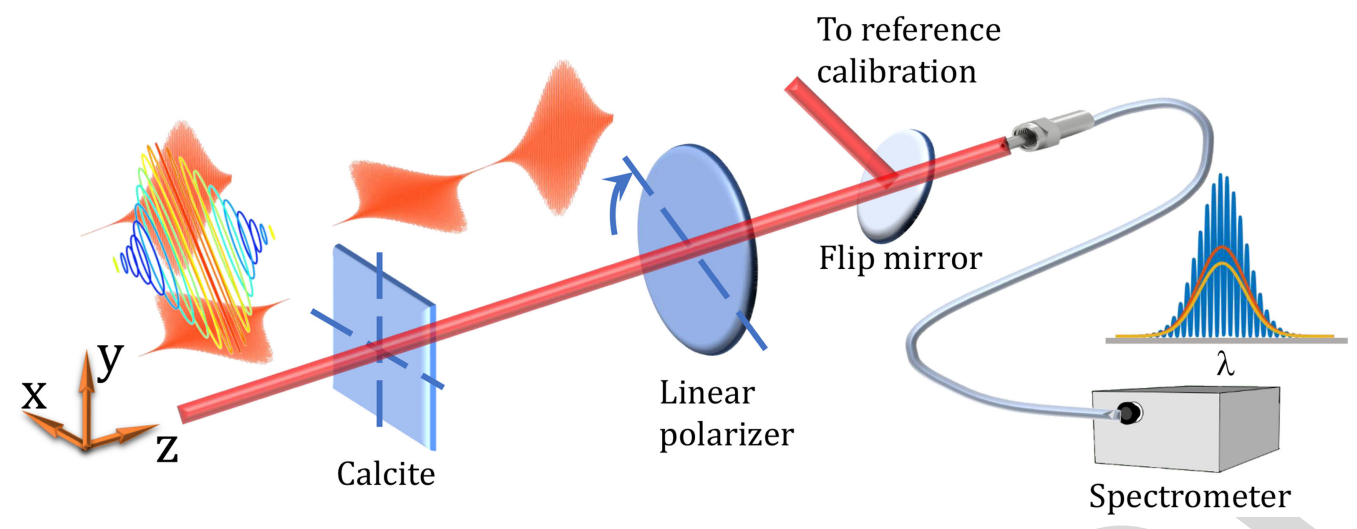

Fig. 1. Schematic of the detection setup for the polarization measurements. The vector pulse passes through a birefringent material (calcite) where the x- and $\mathrm{y}$-components are delayed because of the different dispersion. A linear polarizer selects different projections to be measured in the spectrometer (horizontal and vertical projections for the amplitude; intermediate projection to obtain the relative phase of the pulse components). The spectral phase of the horizontal component is measured with an auxiliary device. The system is calibrated using a pulse linearly polarized at $45^{\circ}$.

interferences. In the case of having LP polarized at $0^{\circ}$ or $90^{\circ}$ (trivial cases), it is possible to rotate the characterization system or the pulse e.g., $45^{\circ}$ to optimize the contrast in the detected fringes (as we do for the large PG case in the next section presenting results). On the other hand, if having different spectra at $0^{\circ}$ and $90^{\circ}$, the same rotation would merge the spectra and lead to fringes in the whole bandwidth.

The technique proposed is multi-shot as it uses 3 individual projections of the calibrating and unknown pulses, plus the reference pulse characterization. The common-path configuration prevents that shot-to-shot fluctuations of the interferometer (due to thermal variations, mechanical vibrations or air fluctuations) affect the measurement. Laser pulse energy fluctuations may affect the retrieval of the relative amplitude of the $\mathrm{x}$ - and $y$-components. In the case of very unstable laser systems, this could be circumvented by using dual-channel spectrometers to simultaneously measure two projections, together with the retrieval of the relative amplitude between $S_{x}$ and $S_{y}$ directly from the intermediate projection $S_{45^{\circ}}$, in a similar way than in [32].

\section{RESULTS: EXPERIMENTS AND SIMULATIONS}

\section{A. Description of the Experiments and Parameters}

For the experiments, we have used a CPA Ti:sapphire laser (Spitfire, from Spectra Physics) at a repetition rate of $1 \mathrm{kHz}$, emitting $100 \mathrm{fs}$ (FWHM) pulses. To select the different spectral projections, we used an achromatic LP (LPVIS050, from Thorlabs). For the simulations, we have used the actual experimental spectrum of the pulse and then calculated the corresponding polarization shaping for the different cases. Notice that our pulse is centered at $797 \mathrm{~nm}$, while the wave plates employed here are designed for $800 \mathrm{~nm}$.

From the experimental characterization, we obtain the temporal amplitude and phase of the vector pulse, from which we calculate the polarization state as a function of frequency and time, presenting the following magnitudes in the results of Fig. 2, 4, 5: spectrum (S) and intensity (I) for $\mathrm{x}$ and y components, ellipticity $\varepsilon=b / a$ calculated as the minor to major axes ratio, phase difference $\delta$ between the $\mathrm{x}$ and y components, and ori- 222 entation of the polarization ellipse (azimuthal angle $\chi$ ) referred 223 to the $\mathrm{x}$-axis. All vector pulse measurements have been done 224 10 times, then calculating the statistical error as the standard 225 deviation and representing it in all the retrieved magnitudes as 226 a gray shaded area (when not seen it means that it is so small 227 that it is not distinguished from the mean value).

\section{B. Validation and Stability Study}

The performance of the technique has been tested at known 230 cases, trivial and non-trivial. For example, the laser pulses have 231 passed through an achromatic half-wave plate, in order to rotate 232 their constant polarization direction, which has been verified in 233 the measurements. Also, we have characterized the input LP 234 pulse passing through a zero-order quarter-wave plate at $45^{\circ} 235$ creating constant circular polarization. 236

Then, a first non-trivial validation consisted in the measure- 237 ment of a pulse linearly polarized at $0^{\circ}$, after passing through a 238 multiple-order quarter-wave plate (QWPM-800-10-4 from CVI, 239 which we label QWPM in the whole manuscript) with the slow 240 axis at $45^{\circ}$. This element consisted of a $652-\mu \mathrm{m}$ thick quartz 241 plate, which introduces a delay of around $20 \mathrm{fs}$ between the fast 242 and slow axes for $800 \mathrm{~nm}$.

We have calibrated it by studying the spectrum transmitted 244 when the plate is placed at $45^{\circ}$ between two crossed linear polar- 245 izers, using as light source both spectrally broadened pulses and 246 incoherent white light. By rotating the last polarizer, clearly the 247 plate behaves as a quarter-wave plate around $800 \mathrm{~nm}$, half-wave 248 plate at $775 \mathrm{~nm}$ and full-wave plate at $825 \mathrm{~nm}$, all at multiple- 249 order regime. Thus, when the light passes through the plate, 250 oriented at $45^{\circ}$, the state of polarization becomes time-varying 251 due to the delay between ordinary and extraordinary compo- 252 nents, being not negligible compared to the pulse duration. 253

To simulate the vector pulse, we have calculated the disper- 254 sion introduced by the QWPM thickness imposing the phase 255 difference $\pi / 2$ at $800 \mathrm{~nm}$. The QWPM angle used in the sim- 256 ulations is $44^{\circ}$ (notice that this is compatible with the experi- 257 mental error in our rotation mount), being the value for which 258 

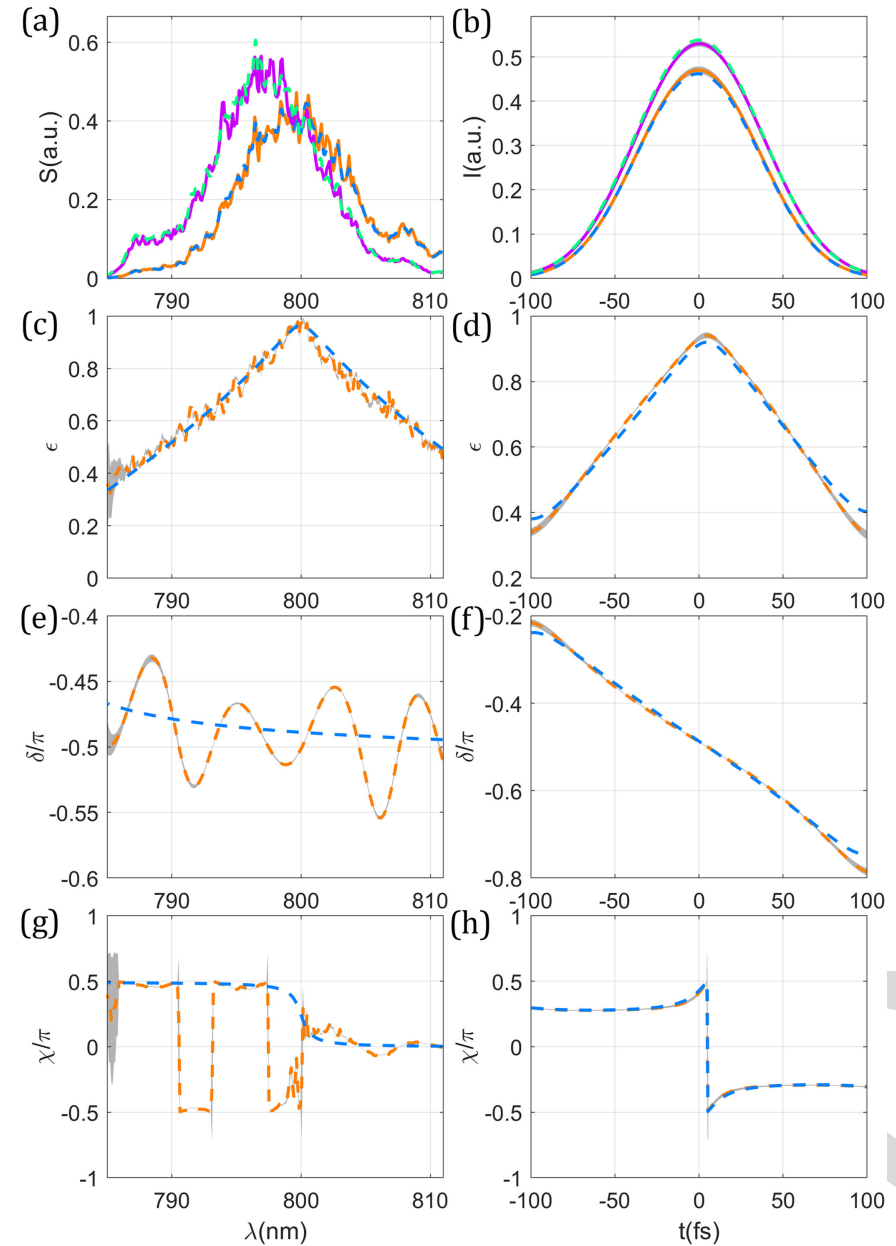

(h)

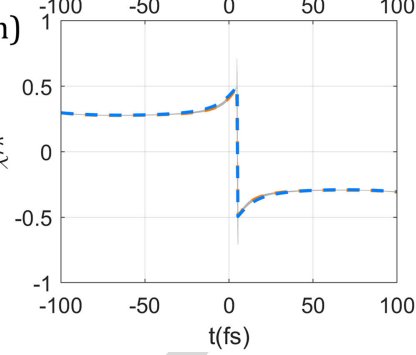

Fig. 2. Polarization results for the case input LP $0^{\circ}$, followed by QWPM slow axis at $45^{\circ}$. Spectral/temporal (a/b) intensity, (c/d) ellipticity $\varepsilon$, (e/f) phase difference $\delta$, and $(\mathrm{g} / \mathrm{h})$ azimuth $\chi$, respectively. Plots (a, b), experimental $x$ (solid red) and $y$ (solid magenta), simulated $x$ (dashed blue) and $y$ (dashed green) components. Plots (c-h), experimental (dashed red), simulated (dashed blue). In all plots the gray shaded area stands for the experimental error.

the simulations match the experimental results $(\varepsilon, \delta$, and $\chi$, simultaneously). Fig. 2 shows the vector pulse reconstruction and simulation results. The spectral amplitude $\mathrm{x}, \mathrm{y}$ components are laterally shifted (due to the effect of dispersion in a multi-order plate), the spectral ellipticity reaches a maximum $(\varepsilon \lesssim 1)$ with circular polarization $(\mathrm{CP})$ at $800 \mathrm{~nm}$, while the peak at the ellipticity depending on time (close to circular polarization) is shifted to positive times with respect to the peak intensity (we have corroborated that this is because the angle of $44^{\circ}$ instead of $45^{\circ}$ ).

In order to show the stability of the technique, we used the interference fringes for a pulse with linear polarization at $45^{\circ}$. We did two stability studies, a short-term one with 300 measurements, 1 per second during 5 minutes, and a long-term one with 1500 measurements, 1 each 5 seconds during $\gtrsim 2$ hours. The fringes where stable and we did not observe the typical mechanical and thermal drift of standard interferometers, as seen in Fig. 3 for the long-term study. Using a fine analysis with the FTSI algorithm, we found the standard deviation of the phase

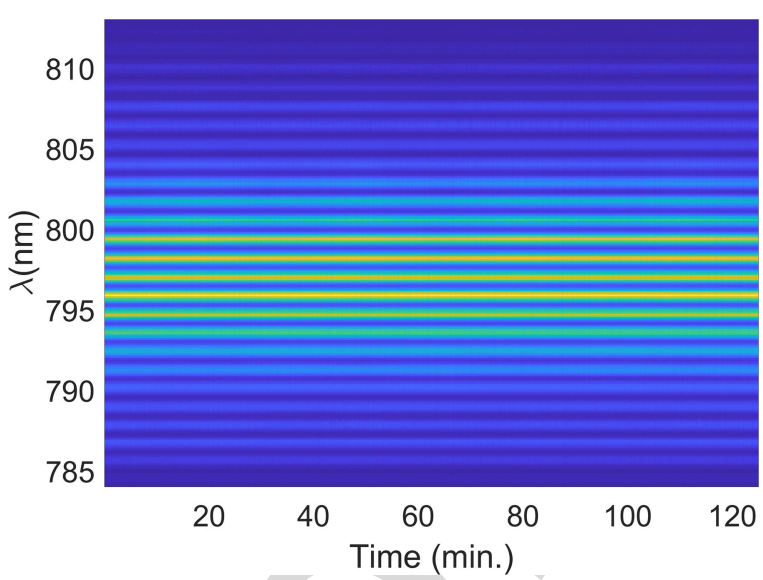

Fig. 3. Spectral interferences recorded with the in-line interferometry setup during 125 minutes used for the long-term stability analysis.

fluctuations to be $9 \cdot 10^{-4} \cdot 2 \pi \mathrm{rad}$ and $4 \cdot 10^{-3} \cdot 2 \pi \mathrm{rad}$ for the 278 short and long-term studies, respectively. Those values are well 279 below the typical values in standard and non-standard interfer- 280 ometers [39]-[42]. The key point for this stability is the in-line 281 geometry that also confers robustness, accuracy and simplicity 282 to the setup. As said before, this is of paramount importance in 283 the case of the determination of the relative phase between the 284 two polarization components, since it is critical for an accurate 285 calculation of the pulse polarization state. Therefore, the setup 286 can be integrated easily and work at environmental conditions 287 less controlled than the present at a laboratory.

\section{Study of Polarization Gates}

Once the performance of the method was tested, we studied 290 the vector pulse coming from PG pulses. As commented pre- 291 viously, that kind of vector pulses are used as one of the main 292 techniques to obtain isolated attosecond pulses [12], [13]. First 293 attempts were performed by using an interferometer in order to 294 delay two orthogonal polarization components of an incoming 295 linearly polarized pulse, in order to obtain circular polarization 296 at the center of the outcoming pulse, and linear polarization at 297 the edges [10]. A zero-order quarter-wave plate (QWP0) aligned 298 at $45^{\circ}$ converted the linear polarization into circular and the cir- 299 cular polarization into linear, obtaining a vector pulse exhibiting 300 linear polarization only in its center, while ellipticity rises at the 301 edges. A simpler and more compact version consists in using 302 a combination of a multiple-order (QWPM) and a zero-order 303 (QWP0) quarter-wave plate [10], [11]. The element QWPM is 304 the same than before, whereas the element QWP0 is a zero-order 305 quartz plate designed for operation at $800 \mathrm{~nm}$ (QWP0-800-08- 306 4-R10 from CVI). The input pulse has LP at $0^{\circ}$, then the QWPM 307 is orientated with the slow axis at $45^{\circ}$. After that, the QWP0 is 308 situated with the fast axis at $90^{\circ}$ to create the so-called narrow 309 PG (results in Fig. 4), characterized by having linear polariza- 310 tion at the center of the pulse and circular polarization at the 311 pulse edges.

After the QWPM, the pulse is that presented in Fig. 2, with 313 $\mathrm{CP}$ at the maximum intensity, with ellipticity decreasing at the 314 

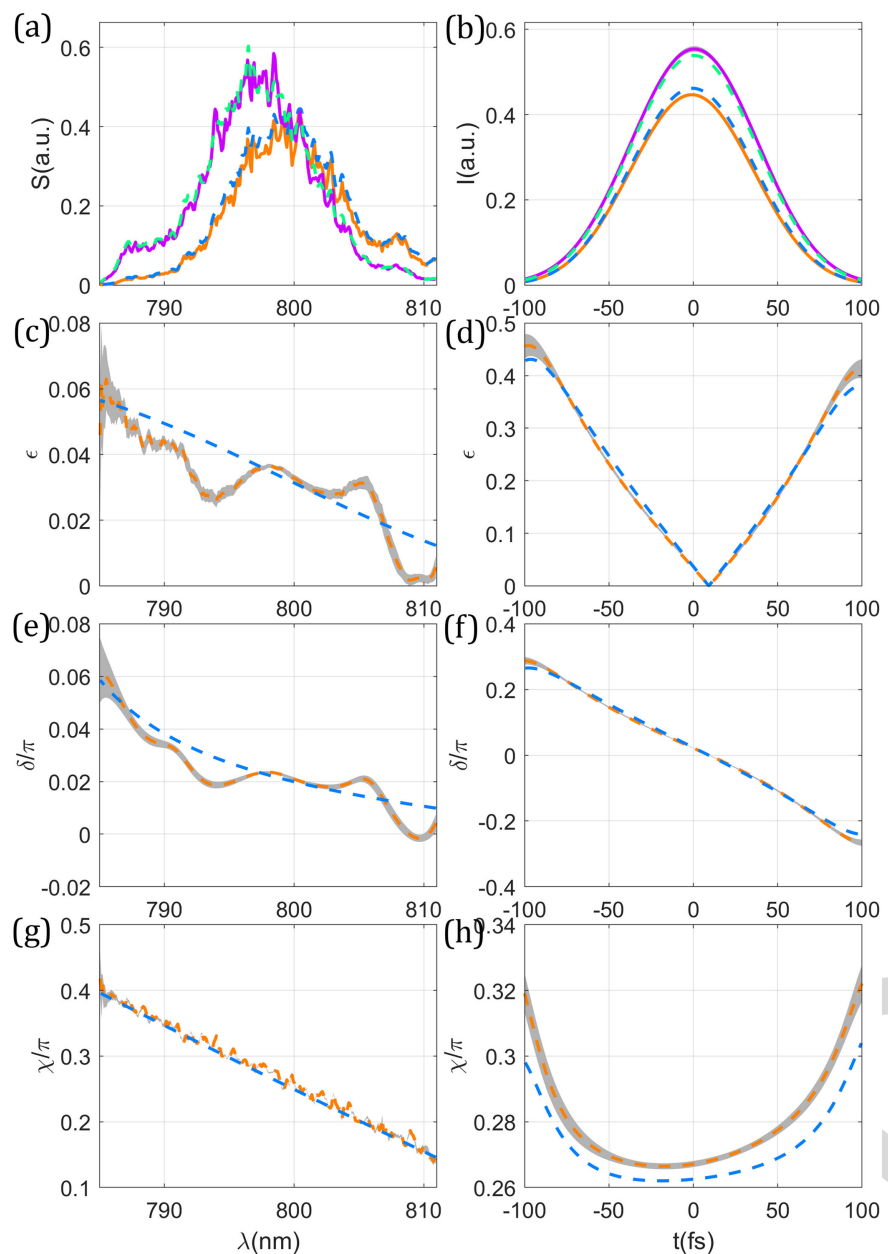

Fig. 4. Polarization results for the narrow PG (input LP $0^{\circ}$, followed by QWPM slow axis at $45^{\circ}$, and QWP0 fast axis at $\left.90^{\circ}\right)$. Spectral/temporal $(\mathrm{a} / \mathrm{b})$ intensity, (c/d) ellipticity $\varepsilon,(\mathrm{e} / \mathrm{f})$ phase difference $\delta$, and $(\mathrm{g} / \mathrm{h})$ azimuth $\chi$, respectively. Plots (a, b), experimental $x$ (solid red) and $y$ (solid magenta), simulated $x$ (dashed blue) and $y$ (dashed green) components. Plots (c-h), experimental (dashed red), simulated (dashed blue). In all plots the gray shaded area stands for the experimental error.

315 edges of the pulse (ideally reaching LP if the delay introduced 316 between the QWPM components is high enough compared to 317 the pulse duration, which is not our case). If then QWP0 is at $90^{\circ}$ 318 (with respect to the $\mathrm{x}$-axis) the $\mathrm{CP}$ of the maximum is converted 319 to LP and the ellipticity at the edges now increases (in the limit 320 ideally being $\mathrm{CP}$ ), thus creating a narrow PG with an effective 321 LP region shorter than the initial pulse duration. The azimuth is 322 almost constant at $45^{\circ}$ in the temporal domain, and the direction 323 of rotation is seen to change at the maximum intensity (with the 324 sign of $\delta$ ).

325 In the so-called large PG, the pulse is characterized because 326 the temporal ellipticity is zero (LP) while the azimuth of the LP 327 is rotating along the pulse duration. The fast axes of the QWP0 328 and QWPM wave plates are aligned at $45^{\circ}$ with respect to the 329 input LP. Under this configuration, the output pulse would be 330 almost $\mathrm{LP}$ at $90^{\circ}$. To avoid this, we rotate the system $45^{\circ}$, where 331 the initial pulse passes through a LP at $45^{\circ}$ (polarizing cube), 332 then the QWPM and the QWP0 wave plates are orientated with
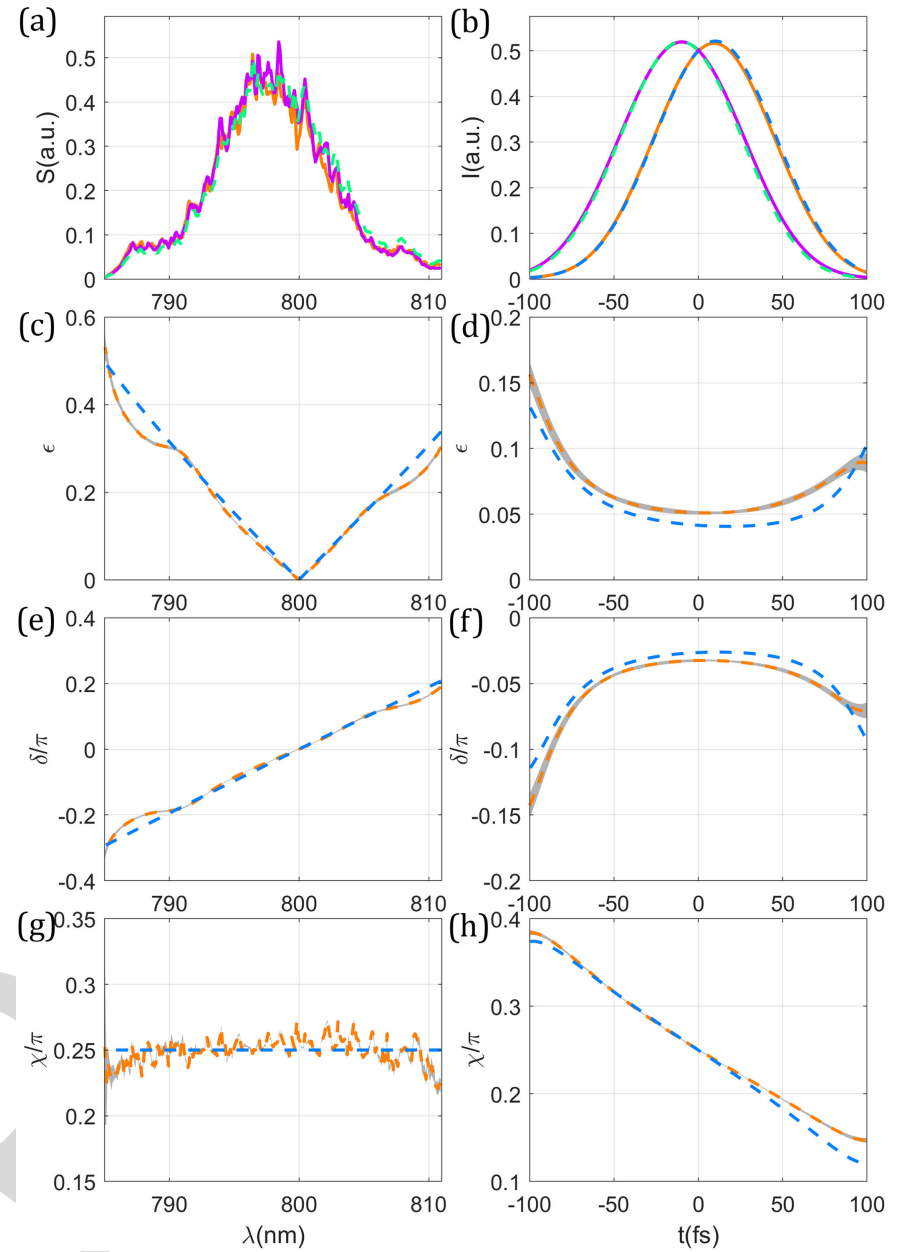

Fig. 5. Polarization results for the large PG (input LP $45^{\circ}$, followed by QWPM fast axis at $0^{\circ}$, and QWP0 fast axis at $\left.90^{\circ}\right)$. Spectral/temporal (a/b) intensity, (c/d) ellipticity $\varepsilon,(\mathrm{e} / \mathrm{f})$ phase difference $\delta$, and (g/h) azimuth $\chi$, respectively. Plots (a, b), experimental $x$ (solid red) and $y$ (solid magenta), simulated $x$ (dashed blue) and $y$ (dashed green) components. Plots (c-h), experimental (dashed red), simulated (dashed blue). In all plots the gray shaded area stands for the experimental error.

their fast axes at $90^{\circ}$ (results in Fig. 5). The temporal ellipticity 333 is kept almost constant below 0.1, LP as expected, while the 334 polarization azimuth evolves on time (from $90^{\circ}$ to $0^{\circ}$, being $45^{\circ} 335$ at the center of the pulse).

A full experimental characterization of the PGs [43] is im- 337 portant to predict, optimize and understand the applications of 338 those pulses [44], considering that the real pulse may differ from 339 the expected one e.g., due to the use of non-ideal retarders or 340 non-Gaussian pulse spectrum.

\section{CONCLUSION}

342

In sum, we have presented a simple and compact in-line, 343 single-channel device to reconstruct vector pulses. The capa- 344 bilities of the technique have been demonstrated with different 345 cases of known pulses, being the results corroborated by the 346 simulations. The small statistical error in the retrievals shows a 347 good performance in the measurements, which is related to the 348 
349 excellent stability of the compact bulk interferometer. We have 350 used it to fully characterize PGs of interest for their associated 351 applications. The robustness of the setup makes it suitable in 352 more demanding future experimental situations, e.g., after non353 linear propagation, out laboratory conditions or to characterize 354 vector beams that are nowadays used in many applications.

\section{REFERENCES}

[1] K. Misawa, "Applications of polarization-shaped femtosecond laser pulses," Adv. Phys. X, vol. 1, no. 4, pp. 544-569, 2016.

[2] S. Kerbstadt et al., "Control of photoelectron momentum distributions by bichromatic polarization-shaped laser fields," New J. Phys., vol. 19, no. 10, 2017, Art. no. 103017.

[3] C. Hernández-García et al., "Extreme ultraviolet vector beams driven by infrared lasers," Optica, vol. 4, no. 5, pp. 520-526, 2017.

[4] Y. Kozawa, D. Matsunaga, and S. Sato, "Superresolution imaging via superoscillation focusing of a radially polarized beam," Optica, vol. 5, no. 2 , pp. 86-92, 2018.

[5] A. L. Smirl, X. Chen, and O. Buccafusca, "Ultrafast time-resolved quantum beats in the polarization state of coherent emission from quantum wells," Opt. Lett., vol. 23, no. 4, pp. 1120-1122, 1998.

[6] Y. Fujimura, L. González, K. Hoki, J. Manz, and Y. Ohtsuki, "Selective preparation of enantiomers by laser pulses: Quantum model simulation for $\mathrm{H}_{2} \mathrm{POSH}$," Chem. Phys. Lett., vol. 306 , no. 1-2, pp. 1-8, 1999.

[7] M. Sato et al., "Terahertz polarization pulse shaping with arbitrary field control," Nat. Photon., vol. 7, no. 9, pp. 724-731, 2013.

[8] S. M. Parker, M. A. Ratner, and T. Seideman, "Simulating strong field control of axial chirality using optimal control theory," Mol. Phys. vol. 110, no. 15-16, pp. 1941-1952, 2012.

[9] H. Rhee et al., "Femtosecond characterization of vibrational optical activity of chiral molecules," Nature, vol. 458, pp. 310-313, 2009.

[10] C. Altucci et al., "Frequency-resolved time-gated high-order harmonics," Phys. Rev. A, vol. 58, no. 5, 1998, Art. no. 3934.

[11] O. Tcherbakoff, E. Mével, D. Descamps, J. Plumridge, and E. Constant, "Time-gated high-order harmonic generation," Phys. Rev. A, vol. 68, no. 4, 2003, Art. no. 043804.

[12] I. J. Sola et al., "Controlling attosecond electron dynamics by phasestabilized polarization gating," Nat. Phys., vol. 2, no. 5, pp. 319-322, 2006.

[13] G. Sansone et al., "Isolated single-cycle attosecond pulses," Science, vol. 314, pp. 443-446, 2006.

[14] C. Hernández-García et al., "Schemes for generation of isolated attosecond pulses of pure circular polarization," Phys. Rev. A, vol. 93, no. 4, 2016, Art. no. 043855.

[15] D. J. Kane and R. Trebino, "Characterization of arbitrary femtosecond pulses using frequency-resolved optical gating," IEEE J. Quantum Electron., vol. 29, no. 2, pp. 571-579, Feb. 1993.

16] C. Iaconis and I. A. Walmsley, "Spectral phase interferometry for direct electric-field reconstruction of ultrashort optical pulses," Opt. Lett., vol. 23, no. 10, pp. 792-794, 1998.

[17] M. Miranda et al., "Characterization of broadband few-cycle laser pulses with the d-scan technique," Opt. Express, vol. 20, no. 17, pp. 18732-18743, 2012.

[18] B. Alonso et al., "Characterization of sub-two-cycle pulses from a hollow-core fiber compressor in the spatiotemporal and spatiospectral domains," Appl. Phys. B, vol. 112, no. 1, pp. 105-114, 2013.

[19] A. Baltuška, M. Pshenichnikov, and D. Wiersma, "Amplitude and phase characterization of 4.5 -fs pulses by frequency-resolved optical gating," Opt. Lett., vol. 23, no. 18, pp. 1474-1476, 1998.

[20] T. Witting et al., "Characterization of high-intensity sub-4-fs laser pulses using spatially encoded spectral shearing interferometry," Opt. Lett., vol. 36, no. 9, pp. 1680-1682, 2011.

[21] M. Canhota, F. Silva, R. Weigand, and Helder M. Crespo, "Inline self-diffraction dispersion-scan of over octave-spanning pulses in the single-cycle regime," Opt. Lett., vol. 42, no. 15, pp. 3048-3051, 2017.
[22] F. Silva et al., "Strategies for achieving intense single-cycle pulses with 418 in-line post-compression setups," Opt. Lett., vol. 43, no. 2, pp. 337-340, 419 2018.

[23] W. J. Walecki, D. N. Fittinghoff, A. L. Smirl, and R. Trebino, "Charac- 421 terization of the polarization state of weak ultrashort coherent signals by 422 dual-channel spectral interferometry," Opt. Lett., vol. 22, no. 2, pp. 81-83, 423 1997.

[24] H. Rhee, J.H. Ha, S.J. Jeon, and M. Cho, "Femtosecond spectral interfer- 425 ometry of optical activity: Theory," J. Chem. Phys., vol. 129, no. 9, 2008, 426 Art. no. 094507.

[25] P. Schlup, O. Masihzadeh, L. Xu, R. Trebino, and R. A. Bartels, "Tomo- 428 graphic retrieval of the polarization state of an ultrafast laser pulse," Opt. 429 Lett., vol. 33, no. 3, pp. 267-269, 2008.

[26] G. E. Jellison and D. H. Lowndes, "Time-resolved ellipsometry mea- 431 surements of the optical properties of silicon during pulsed excimer 432 laser irradiation," Appl. Phys. Lett., vol. 47, no. 7, pp. 718-721, 433 1998.

[27] M. T. Seidel, S. Yan, and H.-S. Tan, "Mid-infrared polarization pulse 435 shaping by parametric transfer," Opt. Lett., vol. 35, no. 4, pp. 478-480, 436 2010.

[28] O. Masihzadeh, P. Schlup, and R. A. Bartels, "Complete polarization state 438 control of ultrafast laser pulses with a single linear spatial light modulator," 439 Opt. Express, vol. 15, no. 26, pp. 18025-18032, 2007.

[29] S. Linden, H. Giessen, and J. Kuhl, "XFROG - A new method for 441 amplitude and phase characterization of weak ultrashort pulses," Phys. 442 Status Solidi B, vol. 206, no. 1, pp. 119-124, 1998.

[30] T. Bauer, S. Orlov, U. Peschel, P. Banzer, and G. Leuchs, "Nanointer- 444 ferometric amplitude and phase reconstruction of tightly focused vector 445 beams," Nat. Photon., vol. 8, no. 1, pp. 23-27, 2014.

[31] A. Rakhman, M.-W. Lin, and I. Jovanovic, "Angle-multiplexed spatial- 447 spectral interferometry for simultaneous measurement of spectral phase 448 and polarization state," Opt. Express, vol. 21, no. 22, pp. 26896-26907, 449 2013.

[32] L. Lepetit, G. Cheriaux, and M. Joffre, "Linear techniques of phase 451 measurement by femtosecond spectral interferometry for applications 452 in spectroscopy," J. Opt. Soc. Am. B, vol. 12, no. 12, pp. 2467-2474, 453 1995.

[33] L. Zheng, O. A. Konoplev, and D. D. Meyerhofer, "Determination 455 of the optical-axis orientation of a uniaxial crystal by frequency- 456 domain interferometry," Opt. Lett., vol. 22, no. 12, pp. 931-933, 457 1997.

[34] C. Dorrer and F. Salin, "Characterization of spectral phase modulation 459 by classical and polarization spectral interferometry," J. Opt. Soc. Am. B, 460 vol. 15 , no. 8 , pp. $2331-2337,1998$.

[35] P. Schlup, J. Wilson, K. Hartinger, and R. A. Bartels, "Dispersion bal- 462 ancing of variable-delay monolithic pulse splitters," Appl. Opt., vol. 46, 463 no. 23 , pp. 5967-5973, 2007.

[36] J. W. Wilson, P. Schlup, and R. Bartels, "Phase measurement of coherent 465 Raman vibrational spectroscopy with chirped spectral holography," Opt. 466 Lett., vol. 33, no. 18, pp. 2116-2118, 2008.

[37] J. W. Wilson, P. Schlup, and R. A. Bartels, "Synthetic temporal aperture 468 coherent molecular phase spectroscopy," Chem. Phys. Lett., vol. 463, 469 no. 4-6, pp. 300-304, 2008.

[38] G. Ghosh, "Dispersion-equation coefficients for the refractive index and 471 birefringence of calcite and quartz crystals," Opt. Commun., vol. 163, 472 no. 1-3, pp. 95-102, 1999.

[39] W. Hou and X. Zhao, "Drift of nonlinearity in the heterodyne interferom- 474 eter," Precis. Eng., vol. 16, no. 1, pp. 25-35, 1994.

[40] M. Holler and J. Raabe, "Error motion compensating tracking interfer- 476 ometer for the position measurement of objects with rotational degree of 477 freedom," Opt. Eng., vol. 54, no. 5, 2015, Art. no. 054101.

[41] P. Bowlan and R. Trebino, "Using phase diversity for the measurement 479 of the complete spatiotemporal electric field of ultrashort laser pulses," 480 J. Opt. Soc. Am. B, vol. 29, no. 2, pp. 244-248, 2012.

[42] B. Alonso et al., "Frequency resolved wavefront retrieval and dynamics 482 of diffractive focused ultrashort pulses," J. Opt. Soc. Am. B, vol. 29, no. 8, 483 pp. 1993-2000, 2012.

[43] M.-W. Lin and I. Jovanovic, "Single-shot measurement of temporally- 485 dependent polarization state of femtosecond pulses by angle- 486 multiplexed spectral-spatial interferometry," Sci. Rep., vol. 6, 2016, 487 Art. no. 32839.

[44] I. J. Sola et al. "Temporal and spectral studies of high-order harmon- 489 ics generated by polarization-modulated infrared fields," Phys. Rev. A, 490 vol. 74, no. 1, 2006, Art. no. 013810. 


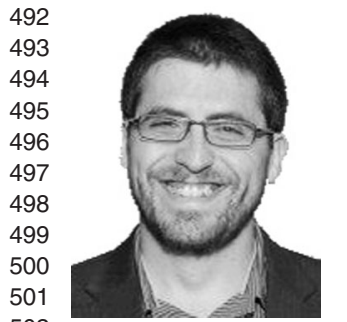

Benjamín Alonso received the B.S. degree in physics in 2007, and the M.S and Ph.D. degrees in physics and technology of lasers in 2008 and 2012, respectively, from the University of Salamanca, Salamanca, Spain.

In 2007, he earned a summer fellowship with the Insitute of Optics, CSIC, Madrid, Spain. From 2008 to 2012, he held a Predoctorate Scholarship with the University of Salamanca and after that he was a Postdoctoral Fellow with the University of Porto, Porto, Portugal. From 2013 to 2016, he was an Instructor 3 with the European College of Aeronautics, Matacán, Spain. From 2014 to 2018, 504 he was an Assistant Professor with the Department of Applied Physics, Poly505 technic High School of Zamora, University of Salamanca. From 2018 to 2019, 506 he was a Postdoctoral Researcher with the Sphere Ultrafast Photonics, Portugal, 507 spin-off of the University of Porto, thanks to a Marie Sklodowska-Curie Indi508 vidual Fellowship. He is currently an Assistant Professor with the University 509 of Salamanca. His main research interests are the characterization of ultrashort 510 laser pulses in the temporal and the spatiotemporal domains, the measurement 511 and applications of the polarization of vector beams, the postcompression of 512 high energy laser pulses, and the optimization of the generation of high-order 513 harmonics.

514 He served the Optical Society of America (OSA) as a Ph.D. Student from 5152009 to 2013 through the OSAL Student Chapter (Salamanca, Spain), which 516 won the Excellence Award from the OSA. He received the Master Award from 517 Foundation "la Caixa" (2008) and the Extraordinary Doctoral Award from the 518 University of Salamanca (2013). He won the Robert S. Hilbert Memorial and 519 was finalist of Emil-Wolf prize from the OSA (Rochester, USA, 2012).

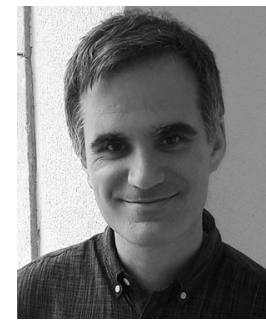

Íñigo J. Sola received the degree in physics in 1998521 and the Ph.D. degree, working on rare earth-doped 522 optical fiber lasers, from the University of Zaragoza, 523 Zaragoza, Spain, in 1998 and 2003, respectively. 524

From 2003 to 2005, he was a Postdoctoral Re- 525 searcher with the Centre Lasers Intenses et Appli- 526 cations (CELIA), Talence, France, in the frame of a 527 European network. His main research interest turned 528 on ultrashort laser pulses. In 2005, he obtained a 529 postdoctoral grant, becoming Associate Professor in 530 2011, both at the University of Salamanca, Spain. His 531 current research interests are focused on ultrafast optics and photonics, including 532 ultrashort pulse control and characterization in the few-cycle regime, nonlinear 533 light-matter interaction, high-order harmonic generation, and their applications. 534 